DOI: 10.12731/2227-930X-2021-11-3-7-34

УДК 504.6:656.13(052)

\title{
ФОРМИРОВАНИЕ УСТОЙЧИВОЙ ТРАНСПОРТНОЙ СИСТЕМЫ НА ОСНОВЕ ФУНКЦИОНАЛЬНОЙ МОДЕЛИ «СРЕДА - УЧАСТНИК - ТРАНСПОРТ - ИНФРАСТРУКТУРА»
}

\section{Загидуллин P.P.}

На состояние и функиионирование транспортной системь влияют многочисленные факторы. Представлен результат анализа работ известных отечественных и зарубежных учёных в области исследования транспортных прочессов. Существующая классическая схема взаимодействия элементов не даёт представления о нахождении пешехода и пассажира в данной системе. $C$ иелью всестороннего изучения взаимодействия всех элементов транспортной системы предлагается использовать систему «среда - участник - транспорт - инфраструктура». Изучение взаимодействия элементов данной подсистемы позволит учесть влияние ключевого человеческого фактора, на состояние транспортных прочессов. Также рассмотрен и обоснован предлагаемый концептуальный алгоритм формирования устойчивой транспортной системы на основе оптимизации совокупных затрат функиионирования модели «среда - участник - транспорт - инфраструктура». Полученные результаты будут полезны руководителям, ответственных за принятие решений в области устойчивого развития и функиионирования транспортной системьл.

Цель-определение способов и приемов анализа, применяющих при проведении исследований функиионирования транспортной системьл.

Метод или методология проведения работы. $B$ статье использовались экономические, экологические и математические методbl. 
Результаты. Получены наиболее информативные параметры, показывающие некоторые аспекты функиионирования транспортной системы.

Область применения результатов: полученные результаты целесообразно применять руководителям, ответственных за принятие решений в области устойчивого развития и функичионирования транспортной системы.

Ключевые слова: безопасность дорожного движения; организачия дорожного движения; система «водитель - автомобильдорога - среда»; система «среда-участник-транспорт-инфраструктура»; транспортная система; дорожно-транспортная инфраструктура; окружающая среда; участник дорожного движения; дорожно-транспортные происшествия; интеллектуальная транспортная система; устойчивая транспортная система

\section{FORMATION OF A SUSTAINABLE \\ TRANSPORT SYSTEM BASED ON THE FUNCTIONAL MODEL «ENVIRONMENT - PARTICIPANT - TRANSPORT - INFRASTRUCTURE»}

\section{Zagidullin R.R.}

Numerous factors influence the condition and functioning of the transport system. The result of the analysis of the works of well-known domestic and foreign scientists in the field of research of transport processes is presented. The existing classical scheme of interaction of elements does not give an idea of the presence of a pedestrian and a passenger in this system. In order to comprehensively study the interaction of all elements of the transport system, it is proposed to use the «environment - participant - transport - infrastructure» system. The study of the interaction of the elements of this subsystem will allow us to take into account the influence of the key human factor on the state of transport processes. The proposed conceptual algorithm for the formation of a sustainable transport system based on the op- 
timization of the total costs of the functioning of the "environmentparticipant-transport-infrastructure» model is also considered and justified. The results obtained will be useful to managers responsible for decision-making in the field of sustainable development and functioning of the transport system.

Purpose. Definition of the ways and receptions of the analysis applying when carrying out the analysis of export operations.

Methodology the article uses economic and mathematical methods, as well as statistical methods of analysis.

Results. The most informative parameters showing some aspects of the functioning of the transport system are obtained.

Practical implications it is advisable to apply the obtained results to managers responsible for decision-making in the field of sustainable development and functioning of the transport system.

Keywords: road safety; traffic management; driver - car - road environment system; environment - participant - transport - infrastructure system; transport system; road transport infrastructure; environment; road user; road accidents; intelligent transport system; sustainable transport system

\section{Введение}

В настоящее время одной из важнейших тенденций городского развития является переход к формированию устойчивых транспортных систем. Это связано с тем, что традиционные подходы к решению проблем перегрузки, только увеличивающие пропускную способность сети, неэффективны. Рост спроса на поездки сосредоточен в определенных частях сети в определенное время. Увеличение пропускной способности сети и строительство новых дорог приводят лишь к временному улучшению. Торговые и промышленные компании строят новые коммерческие и жилые районы, где рядом с новыми дорогами добавляется трафик к дорожной сети. Наконец, через несколько лет эти новые дороги станут перегруженными.

Нужна новая стратегия развития, и она включает стратегию умных городов и городов с устойчивой транспортной системой, обе- 
спечивающая устойчивую мобильность, позволяя перемещаться из одного места в другое. При этом используя мультимодальный подход с самыми высокими стандартами безопасности, предлагая выбор вида транспорта, обеспечивая доступность территорий с различными функциями, ограничивая выбросы и потребление энергии и сводя к минимуму использование земли. Чтобы создать эффективную и устойчивую транспортную систему, город должен изменить транспортную политику с перемещения транспортных средств на перемещение людей и товаров. Это включает в себя учет мультимодальной поездки от места отправления до места назначения, которая, как правило, осуществляется с помощью различных видов транспорта.

\section{Метод системного подхода}

Для изучения групп взаимосвязанных факторов, влияющих на формирование и развитие модели устойчивой транспортной системы, проведены исследования с точки зрения теории системного подхода к деятельности по организации и безопасности дорожного движения, а также индикаторов функционирования транспортной системы (рис. 1). В ходе исследования также выявлены современные предпосылки, причины и стимулы, влияющие на выработку современных требований к комплексному развитию транспортной системы, транспортной инфраструктуры, дорожного движения. Это позволяет предложить новую модель взаимодействующих подсистем, как базовую для формирования комплекса требований к транспортной системе в современных условиях.

Для исследования функционирования транспортной системы предлагается использовать системный подход, который является наиболее эффективным для изучения проблемы. Использование системного подхода при изучении транспортного процесса, а также безопасности дорожного движения (БДД) показало, что ДТП является следствием сочетания ряда причин, из которых трудно выделить главную или оценить роль каждой из них, так как БДД обеспечивается надёжным функционированием всей транспортной системы. 


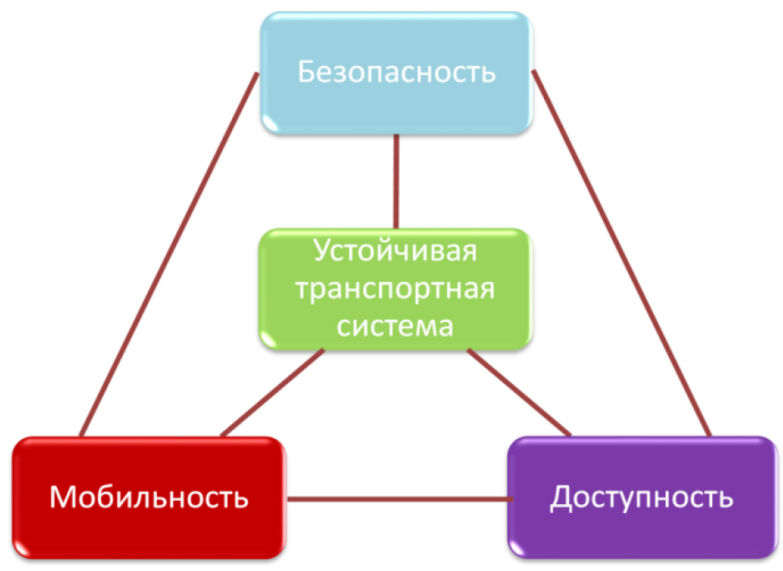

Рис. 1. Модель устойчивой транспортной системы

Для решения проблемы эффективности функционирования транспортной системы и повышения БДД необходимо определить местонахождение участников движения в системе В-А-Д-С на основе анализа исследований отечественных и зарубежных учёных (рис. 2) [1-11].

Следует отметить, что большинство авторов человеческий фактор как взаимодействующей части системы В-А-Д-С используют водителя (В) - считается, что примерно 90\% ДТП происходит по причине несоблюдения именно водителем Правил дорожного движения (ПДД) - и система выглядит как В-А-Д-С [1].

Считается, что первый самый значимый элемент «В - водитель» включает ошибки водителей, вызванные недостаточной профессиональной подготовкой, возрастом, состоянием здоровья, а также темпераментом, результатом которого будет склонность к риску, эмоциональная неустойчивость и т. д. Второй элемент «А - автомобиль» связан с техническим состоянием транспортных средств (ТС) и возможностью обеспечения активной и пассивной безопасности. Третий элемент «Д - дорога» связана с неудовлетворительными дорожными условиями, которые включают в себя не только состояние проезжей части и обочин, но и несоответствие 
размеров геометрических элементов дорог фактической скорости движения, сочетание элементов плана и профиля дороги и т. д. Четвертый элемент «С - среда» связан с неблагоприятными погодно-климатическими условиями: туман, атмосферные осадки, боковой ветер $[2,3]$.

На БДД оказывает влияние большое количество факторов. Для удобства изучения транспортной системы и причин возникновения ДТП все факторы предлагается условно делить на четыре взаимодействующих части: среда $(\mathrm{C})$, участник (У), транспорт (Т), инфраструктура (И), и рассматривают как элементы единой системы С-У-Т-И.

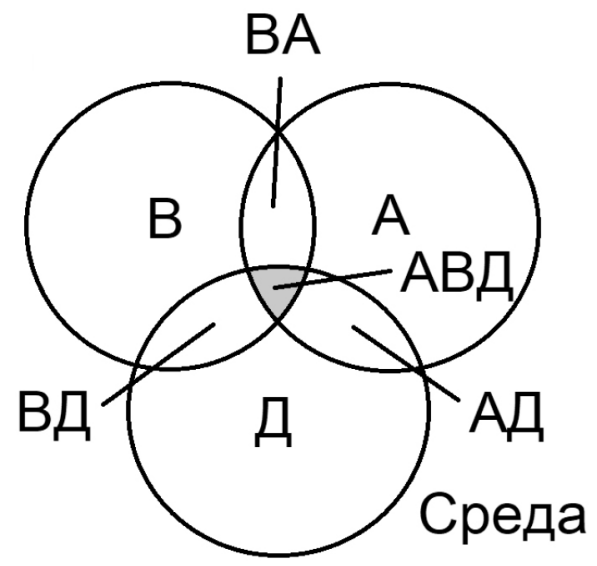

Рис. 2. Система «Водитель - Автомобиль - Дорога - Среда» (В-А-Д-С)

В системе С-У-Т-И основным элементом является «человек» - участник дорожного движения - водитель, пешеход, пассажир. Таким образом, предлагается современная социально-экономически и экологически ориентированная, функционально-технологическая транспортно-инфраструктурная система «Среда - Участник - Транспорт - Инфраструктура» (С-У-Т-И).

Для описания этой системы, оценки и прогнозирования состояния ее подсистем, в зависимости от различных социально-эконо- 
мических и технологических сценариев ее развития, разработана функциональная модель обоснования перечня соответствующих индикаторов и закономерностей (рис. 3).

В модели С-У-Т-И использованы следующие обозначения:

С - окружающая Среда;

У - Участники транспортного процесса (водитель, пешеход, пассажир);

T - Транспорт (все виды транспортных средств, в том числе вспомогательные средства для передвижения маломобильных групп населения с водителем или с автономным управлением);

И - дорожно-транспортная Инфраструктура, учитывающая функциональную классификацию «связь-мониторинг-доступ».

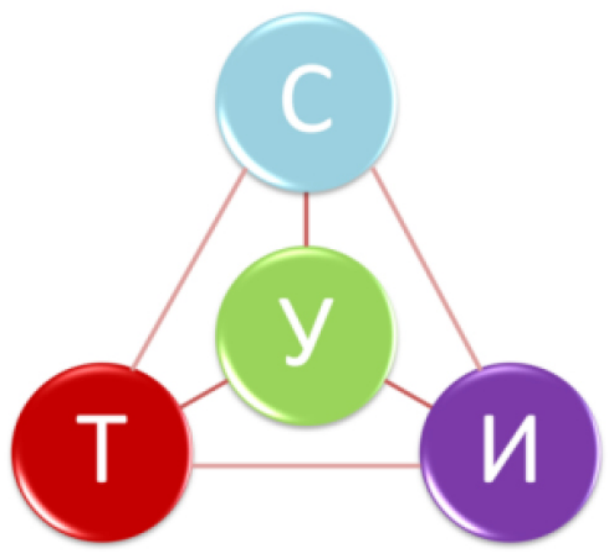

Рис. 3. Система «Среда - Участник - Транспорт - Инфраструктура» (С-У-Т-И)

Важным показателем, характеризующим систему С-У-Т-И, можно считать ее эффективность, а надежность системы С-У-Т-И остается основополагающим фактором оценки ее эффективности. Как и для многих случаев, для оценки эффективности системы С-У-Т-И целесообразным является применение закона сохранения, преобразования и передачи энергии.

Систему С-У-Т-И можно отнести к категории большой и сложной. Она содержит неоднозначные отношения и закономерности 
взаимосвязи между ее элементами. Ввиду многокритериальности системы С-У-Т-И процессы, протекающие в ней, имеют стохастический характер. Следовательно, для данного случая предлагается применять вероятностно-статистические методы оценки эффективности системы С-У-Т-И.

Целью в транспортно-технологическом процессе является эффективное использование транспорта, заключающееся в получении его максимальных динамических качеств, при наименьших затратах энергии. Поэтому целью исследования этой системы является определение того, при каких значениях факторов подсистем можно получить эффективные показатели функционирования транспортной системы для участников дорожного движения.

Вопрос оценки эффективности системы С-У-Т-И мы будем рассматривать на основе методологии системного подхода, которая является объективной необходимостью познания функционирования больших и сложных систем [23].

Существует множество формулировок понятия «система» [24, 25], в соответствии с которыми система С-У-Т-И обладает всеми свойствами системы: целостность, делимость, коммуникативность, динамичность, устойчивость. Поэтому в нашем определении система С-У-Т-И - это совокупность подсистем, находящихся во взаимодействии и образующих целостность, способствующую выполнению работы для удовлетворения потребностей человека. Потребности человек достигаются его целенаправленной деятельностью, выполнением транспортно-технологических работ. При этом основными определяющими моментами являются: 1) управляющая деятельность участников; 2) активные транспортные средства; 3) другие инфраструктурные технические и технологические средства. Система С-У-Т-И охватывает эти моменты (рис. 4).

Системный подход предполагает последовательный переход от общего к частному, выделение объекта исследования из системы для достижения цели исследования. При этом выделяют внутренние и внешние связи, образующие основные признаки системы. Для системы С-У-Т-И такими являются многообразие влияющих фак- 
торов и их стохастичность, зависимость от времени, что приводит к нестационарности системы, и многокритериальность, обуславливающа:

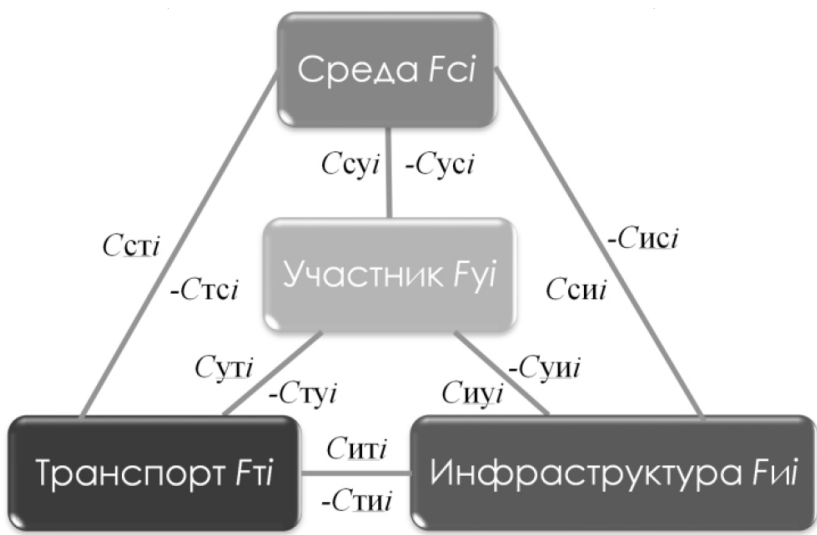
IM [26].

Рис. 4. Функциональная модель формирования экологически и социально-экономически ориентированной, транспортно-инфраструктурной системы С-У-Т-И

Воздействующие на автомобиль факторы:

$C y т i-$ управляющие автомобилем действия водителя, если автомобиль не с автономным управлением;

$C$ ст $i$ - факторы окружающей среды на транспорт-автомобиль;

Ситі - инфраструктурные-дорожные факторы.

На схеме (см. рис. 1) показаны входящие в транспорт-автомобиль, в техническую систему, внешние факторы (связи). Эти факторы, назовем их также сигналами, вызовут ответные реакции элементов технической системы, активируют внутренние связи между ними и сгенерируют выходящие из нее факторы (показатели). Этот процесс можно выразить в операторной форме:

$$
\begin{gathered}
R_{\mathrm{cj}}=S\left(C_{k i}, F_{i}\right), \\
R_{y j}=U\left(C_{k i}, F_{i}\right), \\
R_{m j}=T\left(C_{k i}, Z_{i}\right), \\
R_{u j}=I\left(C_{k i}, Z_{i}\right),
\end{gathered}
$$

где $S, U, T, I$ - операторы подсистем С-У-Т-И; $C_{k i}$ - входящие в подсистему С-У-Т-И факторы; $R_{\mathrm{c},}, R_{y j^{\prime}} R_{m j} R_{u j}$ - выходящие из подси- 
стем С-У-Т-И факторы (расход топлива, загрязняющие вещества и т. д.); $F_{c i} F_{y i}, F_{m i}, F_{u i}$ - внутренние факторы подсистем С-У-Т-И.

Математическая модель динамики подсистемы автомобиль

$$
R_{j}(t)=f\left(C_{k i}(t), F_{i}(t)\right)
$$

Целевая функция оптимизации - это количественная мера экологической, энергетической и экономической оценок

$$
E=f\left(R_{1}, R_{2} \ldots R_{j}\right) .
$$

А транспортно-технологический процесс, выполняемый в системе С-У-Т-И, можно выразить

$$
S_{k}=f(O+E+H) \text {. }
$$

Транспортно-технологический процесс (ТТП) можно охарактеризовать экологическим, энергетическим [27] и экономическим показателями. Так суммарные потери в системе С-У-Т-И на единицу перемещения участника выразятся

$$
S=S_{E}+S_{O}+S_{H}
$$

где $S_{E}$ - энергетические потери; $S_{O}$ - экологические потери; $S_{H}-$ экономические потери.

Решить задачу определения эффективности возможно путем моделирования системы [31]. А выражение (5) может послужить основой концептуальной модели оценки эффективности этой системы.

Ведущим замыслом в системе С-У-Т-И будет, как было сказано в определении системы С-У-Т-И, удовлетворение потребностей человека, т. е. это цель системы, это, согласно выражению (4), выполнение транспортно-технологического процесса. Основной точкой зрения будет потери экологии, энергии и экономики на этот процесс. В выражении (5) показываются все потери.

Поэтому эффективность системы заключается в оптимизации экологического, энергетического и экономического показателей системы С-У-Т-И

$$
\begin{aligned}
& S_{O} \rightarrow \text { min }, \\
& S_{E} \rightarrow \text { min, } \\
& S_{H} \rightarrow \text { min, }
\end{aligned}
$$

где $S_{O}, S_{E}, S_{H}$ - экологические, энергетические и экономические потери в системе С-У-Т-И. 
Формирование устойчивой транспортной системы на основе оптимизации совокупных потерь функционирования модели С-УТ-И представлена на рисунке 5.
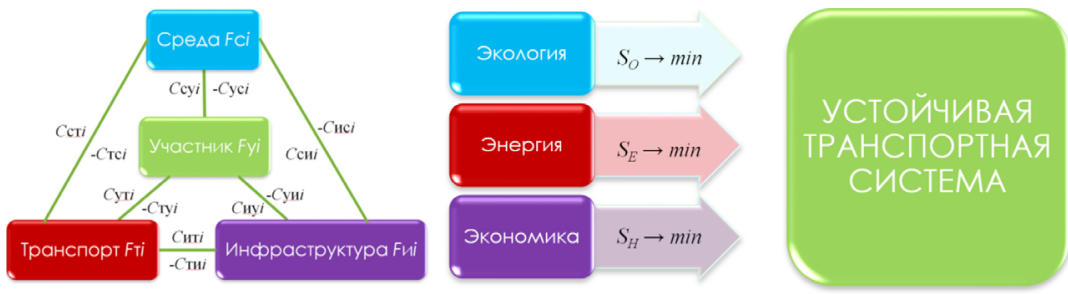

Рис. 5. Функциональная модель формирования устойчивой транспортной системы

План устойчивого развития транспортной системы и городской мобильности нацелен на решение следующих вопросов:

Общественный транспорт. План должен предусматривать стратегию повышения качества, безопасности, интеграции и доступности услуг общественного транспорта, включая всю инфраструктуру, подвижной состав и услуги.

Немоторизованный транспорт. План должен повысить привлекательность и безопасность пешеходного и велосипедного движения. Оценка существующей инфраструктуры с целью ее модернизации там, где это необходимо. Развитие новой инфраструктуры должно быть предусмотрено не только вдоль маршрутов движения моторизированного транспорта. Следует рассмотреть вопрос о создании специальной инфраструктуры для велосипедистов и пешеходов, с тем чтобы обособить их движение от интенсивного моторизованного движения и, при необходимости, сократить дальность маршрутов. Меры, принимаемые в отношении инфраструктуры, должны дополняться другими техническими, а также политическими и «мягкими мерами».

Интермодальность. План должен способствовать более эффективной интеграции различных видов транспорта и предусматривать конкретные меры, направленные на содействие беспрепятственной и мультимодальной мобильности и передвижению. 
Безопасность дорожного движения. План должен включать в себя мероприятия по повышению безопасности дорожного движения на основе анализа основных проблем и зон риска.

Автомобильный транспорт (подвижный и стационарный). План должен учитывать дорожное движение и стоянки транспортных средств в вопросах, связанных с дорожной сетью и моторизованным транспортом. Принимаемые меры должны быть направлены на оптимизацию использования существующей дорожной инфраструктуры и улучшение ситуации как в выявленных проблемных зонах, так и на улучшение общей ситуации. Следует изучить возможность перераспределения дорожного пространства для его использования другими видами транспорта или для выполнения других общественных функций и задач, не связанных с транспортом.

Логистика. План должен включать меры по повышению эффективности городской логистики, включая доставку городских грузов, при одновременном сокращении выбросов парниковых газов, загрязняющих веществ и шума.

Управление мобильностью. План должен включать в себя меры по стимулированию перехода к более устойчивым моделям мобильности. В его подготовке следует задействовать гражданское сообщество, работодателей, школы и т. д.

Интеллектуальные транспортные системы. Интеллектуальные транспортные системы применимы ко всем видам транспорта и услугам мобильности (как к пассажирам, так и к грузовым перевозкам), и полезны при разработке стратегий, осуществлению политики и мониторинге каждой из мер, включенных в план.

Исходя из рассмотренного плана, можно выделить индикаторы устойчивого развития транспортной системы, основанного на принципе трех «лифтов»:

1. повышение безопасности;

2. повышение доступности;

3. повышение мобильности.

Для прогнозирования результатов городского развития и развития городского транспорта требуются передовые и эффективные 
методы моделирования. Могут использоваться различные типы моделей:

- Традиционные модели сетевого и транспортного планирования, которые не учитывают последствия землепользования.

- Модели исследований в области политики, которые дают упрощенное представление о городе и помогают пользователям понять, каких результатов поможет достичь та или иная политика.

- Эскизные модели планирования, которые дают представление о ключевых связях между спросом, предложением и землепользованием внутри города на стратегическом уровне без детализации транспортных сетей или характера землепользования.

- Модели транспорта и городского планирования - это интегрированные модели землепользования и транспорта, которые помогают получить четкое представление о транспортных сетях и моделях землепользования, а также их взаимосвязи с акцентом на стратегические вопросы. Данная модель отличается самой сложной структурой.

Модель землепользования и транспорта помогает понять изменения транспортного поведения людей в ответ на изменения в транспортной системе; каким образом эффективность системы будет реагировать на изменения в характере ее использования; как изменится ситуация с транспортной перегруженностью, загрязнением, дорожно-транспортными происшествиями, мобильностью населения; как изменения в землепользовании повлияют на использование транспортной системы и как изменения в стоимости использования транспортной системы повлияют на землепользование.

На практике рекомендуется сочетать математическое моделирование для прогнозирования количественных показателей с качественными подходами к оценке тех показателей, которые не поддаются количественной оценке (напр., «качество жизни»).

Однако использование этих сложных моделей требует значительного объема ресурсов и специальных знаний.

При разработке и внедрении эффективной системы городского планирования необходимо также сосредоточить внимание на следующих трех ключевых аспектах: 
- Сообщества и местные организации, занимающиеся конкретными видами градостроительной деятельности.

- Заинтересованные стороны, чья деятельность, интересы, потребности и ценности напрямую зависят от вопросов городского развития.

- Площадки для взаимодействия заинтересованных сторон и партнерских организаций.

Вовлечение всех заинтересованных сторон требует гармоничных и конструктивных взаимных усилий. В эту деятельность должны быть вовлечены национальные и местные власти, транспортный сектор и сектор здравоохранения, органы охраны окружающей среды и градостроительства, бизнес-структуры и гражданское общество. Требуется как вертикальная, так и горизонтальная интеграция. Вертикальная интеграция начинается на высшем уровне министерств и спускается к низовым структурам и сообществам и организациям гражданского общества, которые играют ключевую роль в политике устойчивого развития и обеспечивают доступ к информации об окружающей среде и повышают осведомленность в то время, как горизонтальная интеграция предполагает скоординированный стратегический подход во всех четырех секторах - транспорт, здравоохранение, окружающая среда и городское планирование.

Сегодня становится очевидно, что политика адаптирования городов к автомобилям не является эффективным средством борьбы с заторами, так как рано или поздно места всем не хватит. Ошибки транспортно-несостоятельной планировки города в условиях массовой автомобилизации прикрыть невозможно.

Анализ ситуации, сложившейся в большинстве городов развитых и развивающихся стран, позволяет проследить ряд причин:

1. В большинстве городов городской транспорт не рассматривается как единое целое ни с функциональной, ни с территориальной точек зрения.

2. Территориальное планирование зачастую никак не связано с транспортным планированием: оценка того, как новая застрой- 
ка и изменение характера землепользования влияют на дорожное движение, проводится крайне редко.

3. Финансовые ресурсы, выделяемые в городах на нужды городского транспорта, зачастую недостаточны по объему, носят непредсказуемый характер и не проходят процесс стратегического планирования.

4. Отсутствие законодательных требований или рекомендаций. У городских властей отсутствует обязательство составлять стратегические планы по развитию устойчивых городских транспортных систем или планы устойчивой городской мобильности и увязывать их с городским бюджетом.

5. Слабый институциональный и технический потенциал в различных функциональных областях по таким направлениям, как:

- Организация дорожного движения во многих городах трактуется в узком смысле и реализована технически слабо:

- отсутствуют стратегические планы, уделяющие особое внимание этим вопросам, вследствие чего в городах редко принимаются меры инженерного обеспечения дорожного движения, направленные на повышение безопасности пешеходного движения и предоставление права приоритетного проезда общественному транспорту

- интеллектуальные транспортные системы (ИТС) недостаточно широко применяются в сфере организации и мониторинга дорожного движения, а также для информирования пассажиров

- системы светофорного регулирования устарели.

- Работа по осуществлению инвестиций в системы скоростного пассажирского транспорта (метро, ЛРТ, БРТ, городские поезда) и интеграции различных видов транспорта (в части планирования маршрутов, информирования пассажиров в реальном режиме времени, внедрения единого билета, сбора платы за проезд и составления расписаний движения) требует значительного улучшения. В ряде городов системы метрополитена имеют очень небольшую протяженность и зачастую слабо интегрированы с другими видами общественного транспорта; на эксплуатационные затраты уходит львиная доля бюджета городов. 
- Недостаточное регулирование спроса на пользование личным автотранспортом на основе применения альтернативных мер (развитие общественного транспорта, устройство перехватывающих парковок, каршеринг, карпулинг, немоторизованные виды передвижения), а также применение различных мер транспортной политики в целях ограничения поездок на индивидуальном автотранспорте в перегруженных частях городов.

- Демонтаж, переносы и закрытия трамвайных путей и линий, закрытие и банкротство предприятий городского электрического транспорта.

6. Разные виды городского пассажирского транспорта слабо взаимоувязаны как между собой, так и с вело- и пешеходным движением, потенциал которых так и не раскрыт и не используется.

7. Объем инвестиций в инновационные технологии, которые могут улучшить управление транспортными потоками и сделать поездки более комфортными и безопасными для пассажиров, ограничен.

Районы со смешанным характером землепользования и развитой сетью внутренних улиц и проездов характеризуются меньшими расстояниями и смещением доли перемещений на немоторизованные виды передвижения. Для сравнения, раздельное землепользование и преобладание скоростных автострад приводят к увеличению количества и протяжённости автомобильных поездок.

Градостроительные меры сами по себе являются не очень эффективными в силу консервативности поведения людей, поэтому они должны дополняться мерами «кнута и пряника», направленными на преодоление консервативности. Однако наличие правильно организованной структуры землепользования является необходимым элементом для эффективной реализации прочих мероприятий, направленных на предотвращение избыточной мобильности. Учитывая тесную связь между градостроительными аспектами и мобильностью, следует уделять гораздо больше внимания, особенно на ранних стадиях, анализу планируемых мероприятий/проектов с точки зрения их возможного влияния на ге- 
нерацию транспортного спроса. Если увеличения транспортного спроса избежать не представляется возможным, то следует рассмотреть возможные альтернативы, предполагающие использование общественного и немоторизованного транспорта.

Создание благоприятных условий для того или иного вида перемещений приводит к росту его привлекательности в глазах потенциальных пользователей, тем самым создавая предпосылки к возникновению эффекта «индуцированного транспортного спроса». Другими словами, «спрос рождает предложение, а предложение - спрос».

Индуцированный транспортный спрос - это дополнительный пиковый транспортный спрос, возникающий из-за расширения и улучшения дорожной инфраструктуры. Он подразделяется на «переадресованные поездки» (смещение перемещений автомобилей по времени и/или по маршрутам следования) и на «индуцированные поездки» (увеличение среднегодового пробега автомобилей).

Расширение дорожной сети сначала снижает заторы, но это приводит к появлению индуцированного трафика, который возрастает до тех пор, пока заторы вновь не увеличатся и не затормозят его. Поэтому предположение о том, что борьба с заторами приводит к экономии времени автомобилистов, неправомерно.

Также несправедливым является утверждение о том, что увеличение мобильности автомобилистов приносит им дополнительную пользу, т.к. в случае индуцированного спроса автомобилисты в основном совершают «вынужденные» поездки, которые они, в принципе, хотели бы избежать. Из-за такого феномена как «индуцированный транспортный спрос», инвестиции в транспортную инфраструктуру могут привести к более высокому общему спросу на поездки. Увеличение пропускной способности существующих дорог или строительство новых представляют собой популярные способы преодоления заторов. Однако опыт показал, что такие меры не сокращают долгосрочный уровень образования заторов.

Обычно, индуцированный транспортный спрос за несколько лет практически нивелирует эффект от расширения транспортной инфраструктуры. В большинстве случаев расширение дорожной 
сети приводит к снижению её общей эффективности, повышает «внешние» транспортные издержки и увеличивает автомобилезависимость населения. С другой стороны, развёртывание системы транспорта общего пользования (ТОП) постепенно набирает эффективность за счёт привлечения большего количества пользователей.

В связи с этим необходимо предусматривать меры по сдерживанию «гипермобильности». Они включают в себя широкий набор мер административного, экономического и информационного характера, направленных на минимизацию негативных последствий в первую очередь «гиперавтомобилизации».

Приведем некоторые из решений для сдерживания «гипермобильности»:

а) организационные: ограничение права владения транспортным средством; ограничение доступа транспортных средств на определённую территорию; разработка транспортных планов (для предприятий, школ, жилых районов и регионов), а также персональное транспортное планирование; работа на дому; интернет-торговля с доставкой товаров на дом; информационные и маркетинговые кампании;

b) экономические: увеличение стоимости владения транспортным средством; увеличение стоимости использования транспортного средства; плата за парковку автомобилей;

с) инфраструктурные: оптимизация сети транспортной инфраструктуры; перераспределение уличного пространства в пользу пешеходов, велосипедистов и общественного транспорта, «успокоение» движения; ограничение мест для парковки автомобилей; ландшафтный дизайн улиц.

Перечень мер транспортной политики, направленных на улучшение условий движения и предотвращение хронических заторов на улично-дорожной сети, всегда связан с ограничениями спроса и должен включать:

- Применение современных методов организации движения в целях наиболее эффективного использования наличных ресурсов улично-дорожной сети. 
- Реконструкцию пересечений в одном уровне.

- Организацию одностороннего движения на всех участках сети, где эта мера будет способствовать повышению системной пропускной способности.

- Введение жестко регулируемого парковочного режима, в первую очередь на улицах.

- Предоставление преимуществ в движении общественному транспорту, в том числе за счет выделения обособленных полос, предоставления приоритетной зеленой фазы на пересечениях в одном уровне.

- Введение парковочных тарифов с почасовой ставкой, направленных на значительное увеличение платы за долгосрочную стоянку, особенно в центре города.

- Введение норм, стимулирующих автовладельцев обзавестись парковочным местом по месту жительства.

- Введение на улицах в жилой застройке ограничений по скорости движения и сквозному проезду.

- Введение улиц совместного использования и движения с ограничением скоростных режимов.

- Введение платы за пользование отдельными участками улично-дорожной сети. Например, организация платного въезда в центр города.

\section{Выводы}

На основе проведённых исследований можно констатировать факт, что в обеспечении БДД участники дорожного движения имеют преобладающее значение. Их действия определяются индивидуальными качествами, отражающими совокупность физиологических и социально-психологических свойств: воспитание, характер, образование, здоровье, темперамент, тип нервной системы и др. Следовательно, в целях повышения состояния безопасности дорожного движения следует применять систему С-УТ-И, которая в полной мере учитывала бы человеческий фактор в подсистеме «Участник». 
Разработана концептуальная функциональная модель системы С-У-Т-И, которая позволит оценить уровень устойчивости транспортных систем.

Полученная модель функционирования транспортной системы позволит проводить сравнительный анализ по оценке эффективности работы систем городской инфраструктуры и определять перспективные направления ее развития, с целью повышения качества транспортного обслуживания населения.

Основываясь на концептуальную функциональную модель, можно утверждать, что чем выше уровень безопасности, доступности и мобильности, тем выше уровень эффективности устойчивой транспортной системы.

\section{Список литературы}

1. Ильина И.Е. «Пешеход» в системе В-А-Д-С как один из аспектов оценки состояния безопасности дорожного движения // Ученые записки Крымского инженерно-педагогического университета. Симферополь, 2021. № 1 (71). С. 259-263. https://doi.org/10.34771/ UZCEPU.2021.71.1.054

2. Сильянов В.В. Транспортно-эксплуатационные качества автомобильных дорог. М.: Транспорт, 1984. 287 с.

3. Бабков В.Ф. Дорожные условия и безопасность движения. М.: Транспорт, 1982. 288 с.

4. Прохоцкий Т.Г. Резервы безопасности дорожного движения. Минск: Полымя, 1989. 211 с.

5. Сиденко В.М. Эксплуатация автомобильных дорог / В.М. Сиденко, С.И. Михович. М.: Транспорт, 1976. 286 с.

6. Васильев А.П. Состояние дорог и безопасность движения автомобилей в сложных погодных условиях. М.: Транспорт, 1976. 224 с.

7. Коноплянко В.И. Основы безопасности дорожного движения. М.: ДОСААФ, 1978. 128 с.

8. Клинковштейн Г.И. Организация дорожного движения : учеб. для вузов / Г.И. Клинковштейн, М.Б. Афанасьев. 5-е изд., перераб. и доп. М.: Транспорт, 2001. 247 с. 
9. Даутов Ф.М. Исследования параметров транспортного потока южной части московского района города Казани в условиях реконструкции транспортной системы / Р.Р. Загидуллин, Ф.М. Даутов // Современные проблемы безопасности жизнедеятельности: настоящее и будущее. Материалы III Международной научно-практической конференции в рамках форума «Безопасность и связь». Казань: ГБУ «Научный центр безопасности и жизнедеятельности». 2014. С. 596-603.

10.Загидуллин Р.Р. Исследование влияния времени реакции водителя на дорожно-транспортную ситуацию на регулируемом пересечении методом имитационного моделирования // Техника и технология транспорта: научный Интернет-журнал. 2019. № S13. C. 56.

11. Иларионов В.А. Экспертиза дорожно-транспортных происшествий: учебник для вузов. М.: Транспорт, 1989. 255 с.

12. Девятов М.М. Эволюция системы ВАДС как предпосылка формирования современных требований к проектированию дорожно-транспортной инфраструктуры сельских муниципальных образований / М.М. Девятов, А.М. Поляков, Е.В. Столетов // Проектирование автомобильных дорог. Сборник докладов 77-й научно-исследовательской конференции МАДИ. Под научной редакцией П.И. Поспелова. 2019. С. 13-24.

13.Буга П.Г. Организация пешеходного движения в городах / П.Г. Буга, Ю.Д. Шелков. М.: Высшая школа, 1980. 232 с.

14. Самойлов Б.С. Организация и безопасность городского движения / Б.С. Самойлов, В.А. Юдин, П.В. Рушевский. М.: Транспорт, 1989. $156 \mathrm{c}$.

15. Ставничий Ю.А. Дорожно-транспортная сеть и безопасность движения пешеходов. М.: Транспорт, 1983. 72 с.

16. Коршаков И.К. Автомобиль и пешеход: анализ механизма наезда. М.: Транспорт, 1988. 142 с.

17. Лукьянов В.В. Безопасность дорожного движения. М.: Транспорт, 1983. $262 \mathrm{c}$.

18. Ильина И.Е. Выявление и оценка влияния человеческого фактора в возникновении дорожно-транспортного происшествия // Транс- 
порт: наука, техника, управление. Научный информационный сборник. 2020. № 9. С. 59-61.

19. Корчагин В.А. Управление процессами перевозок в открытых социо-природо-экономических автотранспортных системах: монография / В.А. Корчагин, С.А. Ляпин. Липецк: ЛГТУ, 2007. 262 с.

20. Wegman F. Sustainable solutions to improve road safety in the Netherlands / F. Wegman, P. Elsenaar. Research, 1997 (SWOV Report D-097-8). 21 p.

21. Dickinson L. Factor analysis of Pedestrian Accidents / L. Dickinson, I. Hall // Transportation Record. 1976. № 605. P. 35-41.

22. Pedestrian Accident in the USA // HSRJ Research Review. 1982. № 3-4. P. 1-16.

23. Умирзоков А.М. Концептуальная модель оценки эффективности системы «Водитель - Автомобиль - Дорога - Среда» / А.М. Умирзоков, К.Т. Мамбеталин, С.С. Сайдуллозода, А.А. Саибов // Вестник ЮУрГУ. Серия «Машиностроение». 2019. № 1. С. 37-46. https://doi.org/10.14529/engin190104

24. Чернышов В.Н. Теория систем и системный анализ: учеб. пособие / В.Н. Чернышов, А.Н. Чернышов. Тамбов: Изд-во Тамб. гос. техн. ун-та, 2008. 96 с.

25. Haque M. O. Evaluating the effects of the road safety system approach in Brunei / M.O. Haque, T.H. Haque // Transportation Research Part A: Policy and Practice. 2018. Vol. 118. P. 594-607. https://doi. org/10.1016/j.tra.2018.08.017

26. Еремин В.М. Методологические аспекты исследования системы «водитель - автомобиль - дорога - окружающая среда» // Актуальные вопросы повышения безопасности движения: сб. науч. тр. М.: МАДИ, 1988. С. 4-8.

27. Кленин Н.И. Сельскохозяйственные машины / Н.И. Кленин, С.Н. Киселев, А.Г. Левшин. М.: Колос С, 2008. 816 с.

28. Алиев В.А. Надежность тормозных систем автомобилей КамАЗ в специфических условиях республики / В.А. Алиев, А.А. Турсунов // Горизонты науки. 1987. № 4. С. 23-26.

29. Jamous Wael. Assessing travel time reliability implications due to roadworks on private vehicles and public transport services in urban 
road networks / Wael Jamous, Chandra Balijepalli // Journal of Traffic and Transportation Engineering (English Edition). 2018. Vol. 5, iss. 4. P. 296-308. https://doi.org/10.1016/j.jtte.2017.09.003

30.Zhang Rong. Estimation of network level benefits of reliability improvements in intermodal freight transport / Rong Zhang, Wenliang Jian, Lóránt Tavasszy // Research in Transportation Economics. 2018. Vol. 70. Pp. 1-8. https://doi.org/10.1016/j.retrec.2018.09.002

31. Зырянов В.В. Динамическая маршрутизация транспортных потоков как метод снижения транспортной нагрузки на элементы УДС / В.В. Зырянов, А.А. Феофилова, Н.Н. Чуклинов // Мир транспорта и технологических машин. 2018. № 1 (60). С. 74-80.

32. Еремин В.М. Концептуальная модель функционирования системы ВАДС как основа компьютерной имитации // САПР и ГИС автомобильных дорог. 2014. № 1 (2). С. 90-93.

33. Wiese Frauke. Conceptual model of the industry sector in an energy system model: A case study for Denmark / Frauke Wiese, Mattia Baldini // Journal of Cleaner Production. 2018. Vol. 203. P. 427-443. https:// doi.org/10.1016/j.jclepro.2018.08.229.

34. Gent P. A conceptual model for persuasive in-vehicle technology to influence tactical level driver behavior / Paul van Gent, Haneen Farah, Nicole van Nes, Bart van Arem // Transportation Research Part F: Traffic Psychology and Behaviour. 2019. Vol. 60. Pp. 202-216. https:// doi.org/10.1016/j.trf.2018.10.004

35. Kino Yasunobu. Conceptual Modeling supported by Text Analysis / Yasunobu Kino // Procedia Computer Science. 2018. Vol. 126. P. 13871394. https://doi.org/10.1016/j.procs.2018.08.090

\section{References}

1. Il'ina I.E. «Peshekhod» v sisteme V-A-D-S kak odin iz aspektov otsenki sostoyaniya bezopasnosti dorozhnogo dvizheniya ["Pedestrian" in the V-A-D-S system as one of the aspects of assessing the state of road safety]. Uchenye zapiski Krymskogo inzhenerno-pedagogicheskogo universiteta. Simferopol, 2021, no. 1 (71), pp. 259263. https://doi.org/10.34771/UZCEPU.2021.71.1.054 
2. Sil'yanov V.V. Transportno-ekspluatatsionnye kachestva avtomobil'nykh dorog [Transport and operational qualities of highways]. M.: Transport, 1984, $287 \mathrm{p}$.

3. Babkov V.F. Dorozhnye usloviya i bezopasnost'dvizheniya [Road conditions and traffic safety]. M.: Transport, 1982, 288 p.

4. Prokhotskiy T.G. Rezervy bezopasnosti dorozhnogo dvizheniya [Road safety reserves]. Minsk: Polymya, 1989, 211 p.

5. Sidenko V.M., Mikhovich S.I. Ekspluatatsiya avtomobil'nykh dorog [Operation of highways]. M.: Transport, 1976, 286 p.

6. Vasil'ev A.P. Sostoyanie dorog i bezopasnost'dvizheniya avtomobiley $v$ slozhnykh pogodnykh usloviyakh [Road condition and traffic safety in difficult weather conditions]. M.: Transport, 1976, $224 \mathrm{p}$.

7. Konoplyanko V.I. Osnovy bezopasnosti dorozhnogo dvizheniya [Basics of road safety]. M.: DOSAAF, 1978, 128 p.

8. Klinkovshteyn G.I., Afanas'ev M.B. Organizatsiya dorozhnogo dvizheniya : ucheb. dlya vuzov [Organization of traffic]. M.: Transport, 2001, $247 \mathrm{p}$.

9. Dautov F.M., Zagidullin R.R. Issledovaniya parametrov transportnogo potoka yuzhnoy chasti moskovskogo rayona goroda Kazani v usloviyakh rekonstruktsii transportnoy sistemy [Research of the parameters of the traffic flow in the southern part of the Moscow region of the city of Kazan in the context of the reconstruction of the transport system]. Sovremennye problemy bezopasnosti zhiznedeyatel'nosti: nastoyashchee i budushchee. Materialy III Mezhdunarodnoy nauchno-prakticheskoy konferentsii v ramkakh foruma "Bezopasnost' $i$ svyaz'» [Modern problems of life safety: present and future. Materials of the III International Scientific and Practical Conference within the framework of the Security and Communication Forum]. Kazan': GBU «Nauchnyy tsentr bezopasnosti i zhiznedeyatel'nosti», 2014, pp. 596-603.

10.Zagidullin R.R. Issledovanie vliyaniya vremeni reaktsii voditelya na dorozhno-transportnuyu situatsiyu na reguliruemom peresechenii metodom imitatsionnogo modelirovaniya [Investigation of the 
influence of the driver's reaction time on the road traffic situation at a controlled intersection by the method of simulation]. Tekhnika i tekhnologiya transporta, 2019, no. S13, pp. 56.

11. Ilarionov V.A. Ekspertiza dorozhno-transportnykh proisshestviy: uchebnik dlya vuzov [Examination of road traffic accidents: a textbook for universities]. M.: Transport, 1989, 255 p.

12.Devyatov M.M., Polyakov A.M., Stoletov E.V. Evolyutsiya sistemy VADS kak predposylka formirovaniya sovremennykh trebovaniy k proektirovaniyu dorozhno-transportnoy infrastruktury sel'skikh munitsipal'nykh obrazovaniy [Evolution of the VADS system as a prerequisite for the formation of modern requirements for the design of road transport infrastructure of rural municipalities]. Proektirovanie avtomobil'nykh dorog. Sbornik dokladov 77-y nauchno-issledovatel'skoy konferentsii MADI [Design of highways. Collection of reports of the 77th scientific research conference of MADI]. P.I. Pospelov (Ed.), 2019, pp. 13-24.

13. Buga P.G., Shelkov Yu.D. Organizatsiya peshekhodnogo dvizheniya $v$ gorodakh [Organization of pedestrian traffic in cities]. M.: Vysshaya shkola, 1980, $232 \mathrm{p}$.

14. Samoylov B.S., Yudin V.A., Rushevskiy P.V. Organizatsiya i bezopasnost' gorodskogo dvizheniya [Organization and safety of urban traffic]. M.: Transport, 1989, 156 p.

15.Stavnichiy Yu.A. Dorozhno-transportnaya set' $i$ bezopasnost' dvizheniya peshekhodov [Road transport network and pedestrian traffic safety]. M.: Transport, 1983, 72 p.

16.Korshakov I.K. Avtomobil' $i$ peshekhod: analiz mekhanizma naez$d a$ [Car and pedestrian: analysis of the collision mechanism]. M.: Transport, 1988, $142 \mathrm{p}$.

17.Luk'yanov V.V. Bezopasnost'dorozhnogo dvizheniya [Road safety]. M.: Transport, 1983, $262 \mathrm{p}$.

18. Il'ina I.E. Vyyavlenie i otsenka vliyaniya chelovecheskogo faktora v vozniknovenii dorozhno-transportnogo proisshestviya [Identification and assessment of the influence of the human factor in the occurrence of a road traffic accident]. Transport: nauka, tekhni- 
ka, upravlenie. Nauchnyy informatsionnyy sbornik, 2020, no. 9, pp. 59-61.

19.Korchagin V.A., Lyapin S.A. Upravlenie protsessami perevozok v otkrytykh sotsio-prirodo-ekonomicheskikh avtotransportnykh sistemakh: monografiya [Management of transportation processes in open socio-natural-economic transport systems]. Lipetsk: LGTU, 2007, $262 \mathrm{p}$.

20. Wegman F., Elsenaar P. Sustainable solutions to improve road safety in the Netherlands. Research, 1997 (SWOV Report D-097-8). 21 p.

21.Dickinson L., Hall I. Factor analysis of Pedestrian Accidents. Transportation Record, 1976, no. 605, pp. 35-41.

22.Pedestrian Accident in the USA. HSRJ Research Review, 1982, no. 3-4, pp. 1-16.

23.Umirzokov A.M., Mambetalin K.T., Saydullozoda S.S., Saibov A.A. Kontseptual'naya model' otsenki effektivnosti sistemy «Voditel' - Avtomobil' - Doroga - Sreda» [Conceptual model for assessing the effectiveness of the "Driver - Car - Road - Environment" system]. Vestnik YuUrGU. Seriya "Mashinostroenie", 2019, no. 1, pp. 37-46. https://doi.org/10.14529/engin190104

24.Chernyshov V.N., Chernyshov A.N. Teoriya sistem i sistemnyy analiz: ucheb. posobie [Systems theory and systems analysis: textbook. allowance]. Tambov: Izd-vo Tamb. gos. tekhn. un-ta, 2008, 96 p.

25.Haque M.O., Haque T.H. Evaluating the effects of the road safety system approach in Brunei. Transportation Research Part A: Policy and Practice, 2018, vol. 118, pp. 594-607. https://doi.org/10.1016/j. tra.2018.08.017

26.Eremin V.M. Metodologicheskie aspekty issledovaniya sistemy «voditel' - avtomobil' - doroga - okruzhayushchaya sreda» [Methodological aspects of the study of the system "driver - car road - environment"]. Aktual'nye voprosy povysheniya bezopasnosti dvizheniya: sb. nauch. tr. [Actual problems of increasing traffic safety]. M.: MADI, 1988, p. 4-8.

27.Klenin N.I., Kiselev S.N., Levshin A.G. Sel'skokhozyaystvennye mashiny [Agricultural machines]. M.: Kolos S, 2008, 816 p. 
28. Aliev V.A., A.A. Tursunov Nadezhnost' tormoznykh sistem avtomobiley KamAZ v spetsificheskikh usloviyakh respubliki [The reliability of the brake systems of KamAZ vehicles in the specific conditions of the republic]. Gorizonty nauki, 1987, no. 4, pp. 23-26.

29.Jamous Wael, Balijepalli Chandra. Assessing travel time reliability implications due to roadworks on private vehicles and public transport services in urban road networks. Journal of Traffic and Transportation Engineering (English Edition), 2018,.vol. 5, iss. 4, pp. 296-308. https://doi.org/10.1016/j.jtte.2017.09.003

30.Zhang Rong, Jian Wenliang, Tavasszy Lóránt. Estimation of network level benefits of reliability improvements in intermodal freight transport. Research in Transportation Economics, 2018, vol. 70, pp. 1-8. https://doi.org/10.1016/j.retrec.2018.09.002

31.Zyryanov V.V., Feofilova A.A., Chuklinov N.N. Dinamicheskaya marshrutizatsiya transportnykh potokov kak metod snizheniya transportnoy nagruzki na elementy UDS [Dynamic routing of traffic flows as a method of reducing traffic load on the elements of the UDS]. Mir transporta i tekhnologicheskikh mashin, 2018, no. 1 (60), pp. 74-80.

32.Eremin V.M. Kontseptual'naya model' funktsionirovaniya sistemy VADS kak osnova komp'yuternoy imitatsii [Conceptual model of the VADS system functioning as the basis of computer simulation]. SAPR i GIS avtomobil'nykh dorog, 2014, no. 1 (2), pp. 90-93.

33. Wiese Frauke, Baldini Mattia. Conceptual model of the industry sector in an energy system model: A case study for Denmark. Journal of Cleaner Production, 2018, vol. 203, pp. 427-443. https://doi. org/10.1016/j.jclepro.2018.08.229.

34.Gent P., Farah H., Nes N., Arem B. A conceptual model for persuasive in-vehicle technology to influence tactical level driver behavior. Transportation Research Part F: Traffic Psychology and Behaviour, 2019, vol. 60, pp. 202-216. https://doi.org/10.1016/j.trf.2018.10.004

35.Kino Yasunobu. Conceptual Modeling supported by Text Analysis. Procedia Computer Science, 2018, vol. 126, pp. 1387-1394. https:// doi.org/10.1016/j.procs.2018.08.090 


\section{ДАННЫЕ ОБ АВТОРЕ}

Загидуллин Рамиль Равильевич, доцент кафедры «Дорожно-строительных машин», кандидат технических наук Казанский государственный архитектурно-строительнылй университет

ул. Зеленая, 1, г. Казань, Республика Татарстан, 420043, Российская Федеращия r.r.zagidullin@mail.ru

\section{DATA ABOUT THE AUTHOR}

Ramil R. Zagidullin, Associate Professor «Road construction vehicles», Candidate of Technical Sciences Kazan State University of Architecture and Civil 1, Zelenaya Str., Kazan, Republic of Tatarstan, 420043, Russian Federation

r.r.zagidullin@mail.ru

SPIN-code: 7207-8758

ORCID: 0000-0001-5185-2690

ResearcherID: E-5671-2018

Scopus Author ID: 57193743308 